$\operatorname{IPPP} / 06 / 59$

August 2006

\title{
Third-order QCD results on form factors and coefficient functions*
}

\author{
A. Vogt ${ }^{\mathrm{a}}$, S. Moch ${ }^{\mathrm{b}}$ and J.A.M. Vermaseren ${ }^{\mathrm{c}}$ \\ a IPPP, Physics Department, Durham University, South Road, Durham DH1 3LE, United Kingdom

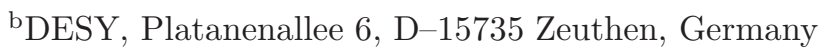 \\ 'NIKHEF, Kruislaan 409, 1098 SJ Amsterdam, The Netherlands
}

We summarize recent higher-order QCD results based, directly or indirectly, on the Mellin-space computation of the next-to-next-to-leading-order splitting functions governing the evolution of hadronic parton distributions. Specifically, we briefly present third-order results for the coefficient functions in inclusive deep-inelastic scattering (including the structure function $F_{3}$ not published so far), the on-shell quark and gluon form factors, and the total cross section for Higgs production at hadron colliders.

\section{Introduction}

For the next decade, the highest-energy experiments in particle physics will be performed at the (anti-)proton-proton colliders TEVATRON and LHC. The cross section for, for example, the inclusive production of a high- $p_{T}$ hadron $h$ at such machines can be schematically written as

$\sigma_{p p \rightarrow h+X}=\sum_{f, f^{\prime}, f^{\prime \prime}} f_{p} * f_{p}^{\prime} * \hat{\sigma}_{\mathrm{ff}^{\prime}}^{\mathrm{f}^{\prime \prime}} * D_{\mathrm{f}^{\prime \prime}}^{h}$.

Here $f_{p}$ stands for the universal momentum distributions of the partons $f$ in the proton $p, f=$ $q_{i}, \bar{q}_{i}, g$ with $i=1, \ldots, n_{f}$, where $n_{f}$ is the number of effectively massless flavours, and $D_{f^{\prime \prime}}^{h}$ are the corresponding fragmentation functions of the final-state hadron $h$. $\hat{\sigma}$ represents the partonic cross section for the process under consideration, and power corrections have been disregarded.

Hence all quantitative collider studies of the standard model, and of expected and unexpected new particles, require a precise understanding of the partonic luminosities and of the QCD corrections to the hard cross sections. For many important processes, like Higgs-boson production, at least the second-order (NNLO) QCD corrections need to be included, i.e., the third term in

$\hat{\sigma}=a_{\mathrm{s}}^{n}\left[\hat{\sigma}^{(0)}+a_{\mathrm{s}} \hat{\sigma}^{(1)}+a_{\mathrm{s}}^{2} \hat{\sigma}^{(2)}+\ldots\right]$.

*Presented by A.V. at Loops and Legs in Quantum Field Theory, 23-28 April 2006, Eisenach (Germany).
The consistent inclusion of $\hat{\sigma}^{(2)}$ requires parton distributions, and in the special case of Eq. (1) also fragmentation functions, evolved with the corresponding NNLO splitting functions

$P_{\mathrm{ff}^{\prime}}^{\mathrm{NNLO}}=a_{\mathrm{s}} P_{\mathrm{ff}^{\prime}}^{(0)}+a_{\mathrm{s}}^{2} P_{\mathrm{ff}^{\prime}}^{(1)}+a_{\mathrm{s}}^{3} P_{\mathrm{ff}^{\prime}}^{(2)}$.

The computation of the third-order splitting functions for the (unpolarized) parton distributions has been completed two years ago [1,2], see also Refs. [3,4]. As briefly recalled below, that calculation was set up such that it could be extended to the third-order coefficient functions in inclusive deep-inelastic scattering (DIS) $[5,6]$. Here we briefly discuss these coefficient functions, as well as some further results based on their calculation [7-9], see also Refs. [10-12]. A discussion of related NNLO results [13] for the evolution of fragmentation functions can be found in Ref. [14].

\section{Third-order DIS coefficient functions}

A calculation of inclusive DIS, $\{\gamma, W\}^{*}(q)+$ $f(p) \rightarrow X$ with $Q^{2} \equiv-q^{2}>0$ and $p^{2}=0$, has been performed in Refs. $[1,2,6]$ up to the third order in the strong coupling $a_{\mathrm{s}}=\alpha_{\mathrm{s}} /(4 \pi)$. The results have been obtained first for all even or odd values of the Mellin variable $N$, via the optical theorem and the three-loop forward Compton amplitudes $\{\gamma, W\}^{*}(q)+f(p) \rightarrow$ $\{\gamma, W\}^{*}(q)+f(p)$. From these expression the complete Bjorken- $x$ space results have been obtained by an automated Mellin inversion $[15,16]$. 
This approach had the crucial advantage of allowing to check the extensive and involved FORM $[17,18]$ codes, at almost any stage, by falling back to the Mincer program $[19,20]$ employed in the previous fixed- $N$ calculations of Refs. [21-23]. An independent check of the $\gamma^{*}$-exchange non-singlet results for $N=16$ has been performed in Ref. [24].

The pole terms of the unfactorized coefficient functions, supplemented by a corresponding calculation of DIS by exchange of a scalar $\phi$ directly coupling to gluons (like the Higgs boson in the heavy top-quark limit), deliver the complete set of NNLO splitting functions [1,2]. The number of relevant diagrams is shown in Table 1 . The finite pieces of the three-loop $\gamma^{*} f$ amplitudes, when calculated also for the $p^{\mu} p^{\nu}$ projection of the hadronic tensor, lead to the $\alpha_{\mathrm{s}}^{3}$ coefficient functions for $F_{L}$ and $F_{2}$ in electromagnetic DIS [5,6].

The former coefficient functions complete the NNLO description of photon-exchange DIS in massless perturbative QCD, see, e.g., Ref. [25]. At large Bjorken- $x$ the latter quantities dominate the $\mathrm{N}^{3} \mathrm{LO}$ corrections for $F_{2}[26,27]$, thus facilitating improved determinations of $\alpha_{\mathrm{s}}$ from data on deep-inelastic scattering. For recent fit-analyses see Ref. [28] and references therein.

\begin{tabular}{lccrrc}
\hline & tree & 1-loop & 2-loop & 3-loop & proj. \\
\hline $\mathrm{q} \gamma$ & 1 & 3 & 25 & 359 & 2 \\
$\mathrm{~g} \gamma$ & & 2 & 17 & 345 & 2 \\
$\mathrm{~h} \gamma$ & & & 2 & 56 & 2 \\
$\mathrm{q} W$ & \multirow{2}{*}{1} & 3 & 32 & 589 & 1 \\
$\mathrm{q} \phi$ & & 1 & 23 & 696 & 1 \\
$\mathrm{~g} \phi$ & 1 & 8 & 218 & 6378 & 1 \\
$\mathrm{~h} \phi$ & & 1 & 33 & 1184 & 1 \\
\hline sum & \multirow{2}{*}{4} & 23 & 394 & 10367 & \\
\hline
\end{tabular}

Table 1

Numbers of diagrams employed in the calculation of the three-loop splitting functions and the corresponding coefficient functions. $h$ denotes the standard ghost used to simplify the gluon polarization sum. The overall sums include the number of Lorentz projections (last column) required for extracting the coefficient functions for $F_{2}$ and $F_{L}$.
The small- $x$ behaviour of the coefficient functions for $F_{2}$ is illustrated in Figs. 1 and 2. The former shows the new $\mathrm{N}^{3} \mathrm{LO}$ contributions $c_{2}^{(3)}(x)$, as in Eq. (2) using the expansion parameter $a_{\mathrm{s}}=$ $\alpha_{\mathrm{s}} /(4 \pi)$, together with successive approximations by the dominant contributions for $x \rightarrow 0$. Even disregarding the additional effect of the ubiquitous Mellin convolution, cf., e.g., Ref. [2], the leading logarithms do not provide a useful approximation in the $x$-range accessible to colliders. In particular, a $20 \%$ accuracy for $c_{2, \mathrm{~ns}}^{(3)}(x)$ is reached with one and two small- $x$ logarithms only at $x<10^{-50}$ (sic) and $x<10^{-14}$, respectively.

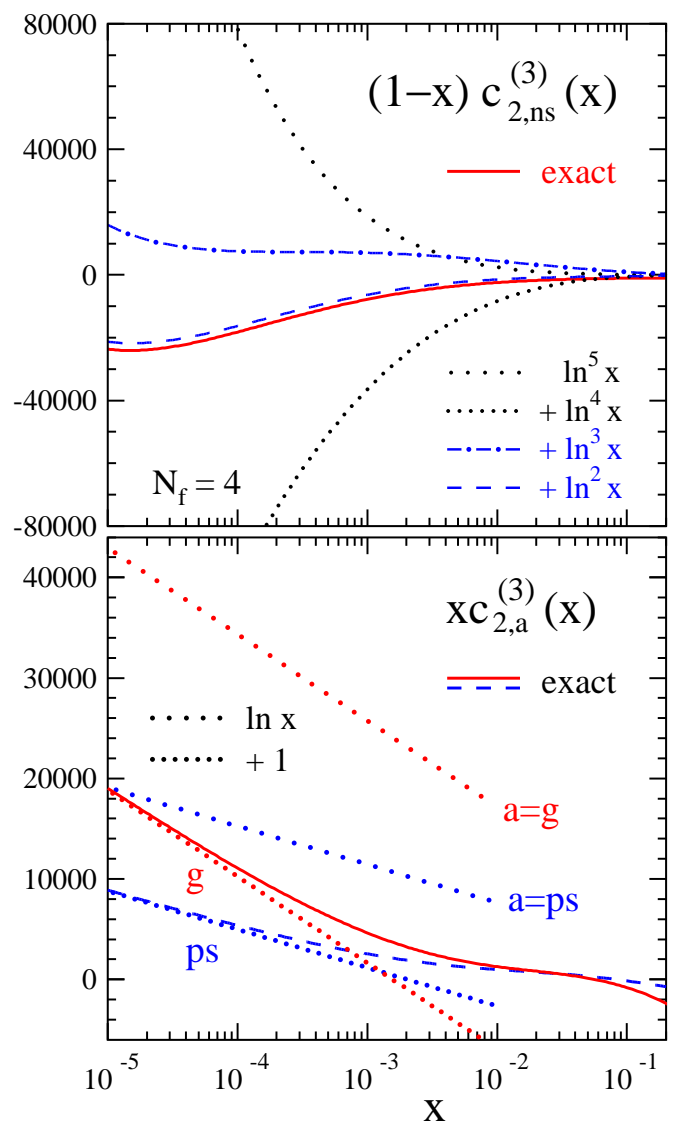

Figure 1. The small- $x$ behaviour of the thirdorder flavour non-singlet (top) and singlet (bottom) coefficient functions for the structure function $F_{2}$ in electromagnetic DIS. Also shown are the respective approximations obtained by successively including the terms leading for $x \rightarrow 0$. 
The perturbative stability at small $x$ is illustrated in Fig. 2 for the gluonic contribution at $Q^{2} \approx 30 \ldots 50 \mathrm{GeV}^{2}$. The third-order correction is far smaller than the second-order contribution first calculated in Ref. [29], exceeding 1\% of the leading-order result only at $x<2 \cdot 10^{-5}$. It should be noted, on the other hand, that the perturbative expansion appears to become unstable at $x \lesssim 10^{-4}$ at very low scales, $Q^{2} \approx 2 \mathrm{GeV}^{2}$ $[5,6]$, due to the larger $\alpha_{\mathrm{S}}$ and especially the flatter small- $x$ shape of the parton distributions.

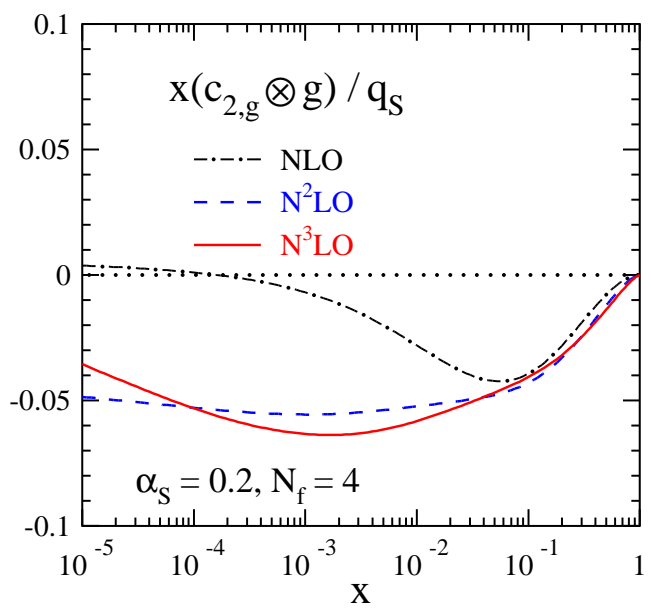

Figure 2. The perturbative expansion up to three loops $\left(\mathrm{N}^{3} \mathrm{LO}\right)$ of the gluon contribution to the flavour-singlet structure function $F_{2}$ for $\alpha_{\mathrm{s}}=0.2$, $n_{f}=4$ and $x g=1.6 x^{-0.3}(1-x)^{4.5}\left(1-0.6 x^{0.3}\right)$. The results have been normalized to the leadingorder result given by the singlet quark distribution $x q_{\mathrm{s}}=0.6 x^{-0.3}(1-x)^{3.5}\left(1+5.0 x^{0.8}\right)$.

The convergence of the perturbation series at large- $x$ / large- $N$ can be conveniently illustrated by displaying the value $\widehat{\alpha}_{a}^{(n)}(N)$ for which the effect of the $n$-th order term $c_{a}^{(n)}(N)$ is half as large as that of $c_{a}^{(n-1)}(N)$. This quantity would be order-independent for a geometric series, while a systematic decrease with increasing $n$ would indicate the asymptotic character of the expansion. $\widehat{\alpha}_{a, \mathrm{~ns}}^{(n)}(N)$ are shown in Fig. 3 for $a=2, L$ (note the different scales). No sign is observed of an imminent breakdown of the perturbative expansion at phenomenologically relevant values of $N$.

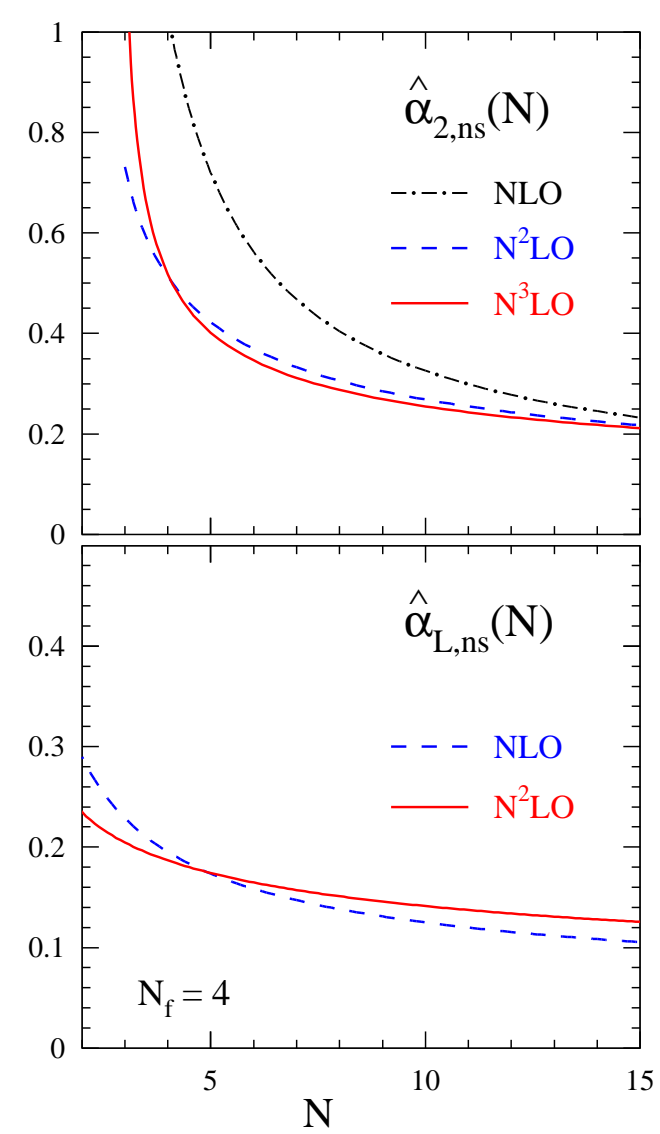

Figure 3. The $N$-dependent values of $\alpha_{\mathrm{s}}$ at which the effect of the $n$-th order $\left(\mathrm{N}^{n} \mathrm{LO}\right.$ for $F_{2}$, $\mathrm{N}^{n-1} \mathrm{LO}$ for $F_{L}$ ) non-singlet coefficient functions is half as large as that of the previous order.

The third-order coefficient function has also been computed for the (odd- $N$ ) non-singlet quantity $F_{3}^{\nu+\bar{\nu}}$, the $q W$ entry in Table 1 . The lengthy exact results and a full discussion will be presented elsewhere [30]. Here we only provide a compact $x$-space parametrization, which is sufficiently accurate for all numerical purposes and which can be easily transformed to Mellin space for use which complex- $N$ codes like Ref. [31]. This parametrization is given in Eq. (4) using the abbreviations $x_{1}=1-x, L_{0}=\ln x, L_{1}=\ln x_{1}$, and $\mathcal{D}_{k}=\left[x_{1}^{-1} L_{1}^{k}\right]_{+}$for the usual +-distributions. The factor $f l_{02}$ (=1 for the numerical evaluation) indicates the $d^{a b c} d_{a b c}$ contribution entering at this order for the first time, cf. Ref. [23]. 


$$
\begin{aligned}
c_{3, \nu+\bar{\nu}}^{(3)}(x) \cong \quad & 512 / 27 \mathcal{D}_{5}-5440 / 27 \mathcal{D}_{4}+501.099 \mathcal{D}_{3}+1171.54 \mathcal{D}_{2}-7328.45 \mathcal{D}_{1}+4442.76 \mathcal{D}_{0} \\
& -9172.68 \delta\left(x_{1}\right)-512 / 27 L_{1}^{5}+8896 / 27 L_{1}^{4}-1396 L_{1}^{3}+3990 L_{1}^{2}+14363 L_{1} \\
& -1853-5709 x+x x_{1}(5600-1432 x)-L_{0} L_{1}\left(4007+1312 L_{0}\right)-0.463 x L_{0}^{6} \\
& -293.3 L_{0}-1488 L_{0}^{2}-496.95 L_{0}^{3}-4036 / 81 L_{0}^{4}-536 / 405 L_{0}^{5} \\
+\quad n_{f} & \left\{640 / 81 \mathcal{D}_{4}-6592 / 81 \mathcal{D}_{3}+220.573 \mathcal{D}_{2}+294.906 \mathcal{D}_{1}-729.359 \mathcal{D}_{0}+2575.46 \delta\left(x_{1}\right)\right. \\
& -640 / 81 L_{1}^{4}+32576 / 243 L_{1}^{3}-660.7 L_{1}^{2}+959.1 L_{1}+516.1+x x_{1}(635.3+310.4 x) \\
& -465.2 x+31.95 x_{1} L_{1}^{4}+L_{0} L_{1}\left(1496+270.1 L_{0}-1191 L_{1}\right)-1.200 x L_{0}^{4}+366.9 L_{0} \\
& \left.+305.32 L_{0}^{2}+48512 / 729 L_{0}^{3}+304 / 81 L_{0}^{4}\right\} \\
+\quad n_{f}^{2} & \left\{64 / 81 \mathcal{D}_{3}-464 / 81 \mathcal{D}_{2}+7.67505 \mathcal{D}_{1}+1.00830 \mathcal{D}_{0}-103.2602 \delta\left(x_{1}\right)-64 / 81 L_{1}^{3}\right. \\
& +992 / 81 L_{1}^{2}-49.65 L_{1}+11.32+51.94 x-x x_{1}(44.52+11.05 x)+0.0647 x L_{0}^{4} \\
& \left.-L_{0} L_{1}\left(39.99+5.103 L_{0}-16.30 L_{1}\right)-16.00 L_{0}-2848 / 243 L_{0}^{2}-368 / 243 L_{0}^{3}\right\} \\
+f l_{02} & n_{f}\left\{2.147 L_{1}^{2}-24.57 L_{1}+48.79-x_{1}(242.4-150.7 x)-L_{0} L_{1}\left(81.70+9.412 L_{1}\right)\right. \\
& \left.+x L_{0}\left(218.1+82.27 L_{0}^{2}\right)-477.0 L_{0}-113.4 L_{0}^{2}+17.26 L_{0}^{3}-16 / 27 L_{0}^{5}\right\} x_{1} .
\end{aligned}
$$

Likewise the (experimentally irrelevant) coefficient functions for DIS via Higgs exchange have been computed to three loops in the heavy topquark limit, see Table 1 . The $x \rightarrow 1$ limit of these (also still unpublished) expressions forms the basis for the Hgg results in the next two sections.

\section{Massless quark and gluon form factors}

The form factors of quarks and gluons are gauge invariant (but infrared divergent) parts of the perturbative corrections to inclusive hard scattering processes. They summarize the QCD corrections to the $q q X$ and $g g X$ vertices with a colour-neutral particle $X$ of either space-like or time-like momentum $q$. These quantities are also key ingredients in the infrared factorization of general higher-order amplitudes $[32,33]$.

The relevant amplitude for the space-like $\gamma^{*} q q$ case, directly entering the photon-exchange coefficient functions discussed above, reads

$\Gamma_{\mu}=\mathrm{i} e_{\mathrm{q}}\left(\bar{u} \gamma_{\mu} u\right) \mathcal{F}_{\mathrm{q}}\left(\alpha_{\mathrm{s}}, Q^{2}\right)$

where $e_{\mathrm{q}}$ represents the quark charge and $Q^{2}=$ $-q^{2}$ the virtuality of the photon. The gaugeinvariant scalar function $\mathcal{F}_{\mathrm{q}}$ is the space-like quark form factor which can be calculated order by order in the strong coupling in dimensional regularization with $D=4-2 \varepsilon$.
The corresponding $H g g$ vertex defining $\mathcal{F}_{\mathrm{g}}$ is an effective interaction in the heavy top-quark limit,

$\mathcal{L}_{\text {eff }}=-\frac{1}{4} C_{H} H G_{\mu \nu}^{a} G^{a, \mu \nu}$,

where $G_{\mu \nu}^{a}$ denotes the gluon field strength tensor, and the coefficient $C_{H}$ includes all QCD corrections to the top-quark loop, known to $\mathrm{N}^{3} \mathrm{LO}[34]$, see also Refs. [35,36].

The quark and gluon form factors were directly calculated at two loops in Refs. [37] and [38] to or$\operatorname{der} \varepsilon^{0}$, respectively, and extended to (all) higher powers of $\varepsilon$ in Refs. [7,8,39]. For the status of a direct three-loop calculation see Ref. [40].

The $\varepsilon^{-6} \ldots \varepsilon^{-1}$ pole terms of the three-loop form factors can be extracted from the thirdorder coefficient functions for DIS $[7,8]$. For this purpose we consider the bare (unrenormalized and unfactorized) partonic structure functions $F^{\mathrm{b}}$ for $\gamma^{*} \mathrm{q} \rightarrow \mathrm{q} X$ and $\phi^{*} \mathrm{~g} \rightarrow \mathrm{g} X$ in the limit $x \rightarrow 1$. Keeping, at each order $\alpha_{\mathrm{s}}^{n}$, only the singular pieces proportional to $\delta(1-x)$ and the + -distributions

$\mathcal{D}_{l}=\left[\frac{\ln ^{l}(1-x)}{(1-x)}\right]_{+}, \quad l=1, \ldots 2 n-1$,

these results are compared to the general structure of the $n$-th order contribution $F_{n}^{\mathrm{b}}$ in terms of 
the $l$-loop form factors $\mathcal{F}_{l}$ and the corresponding real-emission parts $\mathcal{S}_{l}$,

$$
\begin{aligned}
F_{0}^{\mathrm{b}=} & \delta(1-x) \\
F_{1}^{\mathrm{b}}= & 2 \mathcal{F}_{1} \delta(1-x)+\mathcal{S}_{1} \\
F_{2}^{\mathrm{b}}= & \left(2 \mathcal{F}_{2}+\mathcal{F}_{1}^{2}\right) \delta(1-x)+2 \mathcal{F}_{1} \mathcal{S}_{1}+\mathcal{S}_{2} \\
F_{3}^{\mathrm{b}}= & \left(2 \mathcal{F}_{3}+2 \mathcal{F}_{1} \mathcal{F}_{2}\right) \delta(1-x) \\
& +\left(2 \mathcal{F}_{2}+\mathcal{F}_{1}^{2}\right) \mathcal{S}_{1}+2 \mathcal{F}_{1} \mathcal{S}_{2}+\mathcal{S}_{3} .
\end{aligned}
$$

The $x$-dependence of the real emission factors $\mathcal{S}_{k}$ in inclusive deep-inelastic scattering is of the form $\mathcal{S}_{k}\left(f_{k, \varepsilon}\right)$, where the $D$-dimensional +-distributions $f_{k, \varepsilon}$ are defined by

$$
\begin{aligned}
f_{k, \varepsilon}(x) & =\varepsilon\left[(1-x)^{-1-k \varepsilon}\right]_{+} \\
& =-\frac{1}{k} \delta(1-x)+\sum_{i=0} \frac{(-k \varepsilon)^{i}}{i !} \varepsilon \mathcal{D}_{i}
\end{aligned}
$$

Thus, exploiting this particular analytical dependence, the $n$-loop form factor $\mathcal{F}_{n}$ can simply be extracted by the substitution

$\mathcal{D}_{0} \rightarrow \frac{1}{n \varepsilon} \delta(1-x)-\sum_{i=1} \frac{(-n \varepsilon)^{i}}{i !} \mathcal{D}_{i}$,

once the combinations of lower-order quantities in Eq. (8) - determined before to a sufficiently high order in $\varepsilon$ - have been subtracted from the calculated results for $F_{n}^{\mathrm{b}}$. As $\delta(1-x)$ enters with a factor $1 / \varepsilon$, this extraction loses one power in $\varepsilon$. Hence from the third-order calculation to order $\varepsilon^{0}$, as performed for the coefficient functions, only the pole terms of $\mathcal{F}_{3}$ can be obtained in this manner. So far the $\varepsilon^{0}$-term has only been derived for the fermionic $\left(n_{f}\right)$ part of the quark form factor [8], via extending the corresponding DIS calculation [41] by one order in $\varepsilon$.

The reader is referred to Refs. [7,8,11] for a further discussion of these results, including their very interesting structure in the context of the exponentiation of the form factors [42-45]. We would like to note, however, that the $\alpha_{\mathrm{s}}^{3} \varepsilon^{-1}$ coefficients of the highest $\zeta$-function weights, $\zeta_{2} \zeta_{3}$ and $\zeta_{5}$, agree with the results inferred, using the conjecture of Ref. [46], from the calculation in $\mathcal{N}=4$ Super-Yang-Mills theory in Ref. [47].

\section{Higgs productions at (almost) $\mathrm{N}^{3} \mathrm{LO}$}

Armed with the third-order splitting functions $[1,2]$ and the results of the previous section, one can now derive all soft-enhanced (+-distribution) contributions to the $\mathrm{N}^{3} \mathrm{LO}$ cross sections $\hat{\sigma}^{(3)}$ for lepton-pair and Higgs boson production at colliders $[9,50]$, see also Refs. [51,52]. Analogous to Eq. (8), the soft limit of the bare cross sections $W^{\mathrm{b}}$ for $\mathrm{q} \overline{\mathrm{q}} \rightarrow \gamma^{*} \rightarrow l^{+} l^{-}$and $\mathrm{gg} \rightarrow H$ reads

$W_{0}^{\mathrm{b}}=\delta(1-x)$

$W_{1}^{\mathrm{b}}=2 \operatorname{Re} \mathcal{F}_{1} \delta(1-x)+\mathcal{S}_{1}$

$W_{2}^{\mathrm{b}}=\left(2 \operatorname{Re} \mathcal{F}_{2}+\left|\mathcal{F}_{1}\right|^{2}\right) \delta(1-x)+2 \operatorname{Re} \mathcal{F}_{1} \mathcal{S}_{1}$ $+\mathcal{S}_{2}$

$W_{3}^{\mathrm{b}}=\left(2 \operatorname{Re} \mathcal{F}_{3}+2\left|\mathcal{F}_{1} \mathcal{F}_{2}\right|\right) \delta(1-x)$ $+\left(2 \operatorname{Re} \mathcal{F}_{2}+\left|\mathcal{F}_{1}\right|^{2}\right) \mathcal{S}_{1}+2 \operatorname{Re} \mathcal{F}_{1} \mathcal{S}_{2}+\mathcal{S}_{3}$,

where, of course, $\mathcal{F}$ now denotes the time-like quark or gluon form factor, known by analytic continuation from $q^{2}=-Q^{2}<0$ to $q^{2}>0$.

The real-emission contributions $\mathcal{S}_{k}$ depend on the scaling variable $x=M_{\gamma^{*}, H}^{2} / s$. Here the dependence of $\mathcal{S}_{k}$ on $x$ is of the form $\mathcal{S}_{k}\left(f_{2 k, \varepsilon}\right)$, i.e.,

$\mathcal{S}_{k}=f_{2 k, \varepsilon} \sum_{l=-2 k}^{\infty} 2 k s_{k, l} \varepsilon^{l}$.

With the known time-like form factors, the expansion coefficients $s_{k, l}$ of the soft function $\mathcal{S}_{k}$ can be derived recursively as far as they are subject to the KLN cancellations and the mass-factorization structure relating the remaining poles to the splitting functions (3). Employing the results of Refs. [7,8] and [1,2], the third-order terms $s_{3,-6} \ldots s_{3,-1}$ can be obtained. Due to Eq. (9) this is sufficient to derive all +-distribution contributions to the third-order coefficient functions. The explicit results can be found in Ref. [9].

Focusing on Higgs production, we note that a good approximation (to about $10 \%$ or less) to the double convolutions $g * g * \hat{\sigma}$ is obtained at NLO and NNLO [53-55] by transforming to $N$-space and keeping only the $\ln ^{k} N$ and $N^{0}$ terms arising from the +-distributions in $\hat{\sigma}^{(1)}$ and $\hat{\sigma}^{(2)}$. Consequently the above results facilitate a sufficient approximation to the complete $\mathrm{N}^{3} \mathrm{LO}$ correction, with a (conservative) error estimate of $20 \%$. 
The resulting predictions are illustrated for the LHC in Fig.4, where all higher-order contributions have been calculated in the heavy top-quark approximation, but are normalized to the full lowest-order cross sections. Considering these and other results [9], $5 \%$ at the LHC, and $7 \%$ at the Tevatron, appears to represent a conservative estimate of the improved cross-section uncertainty due to the truncation of the perturbation series at the (approximated) $\mathrm{N}^{3} \mathrm{LO}$.
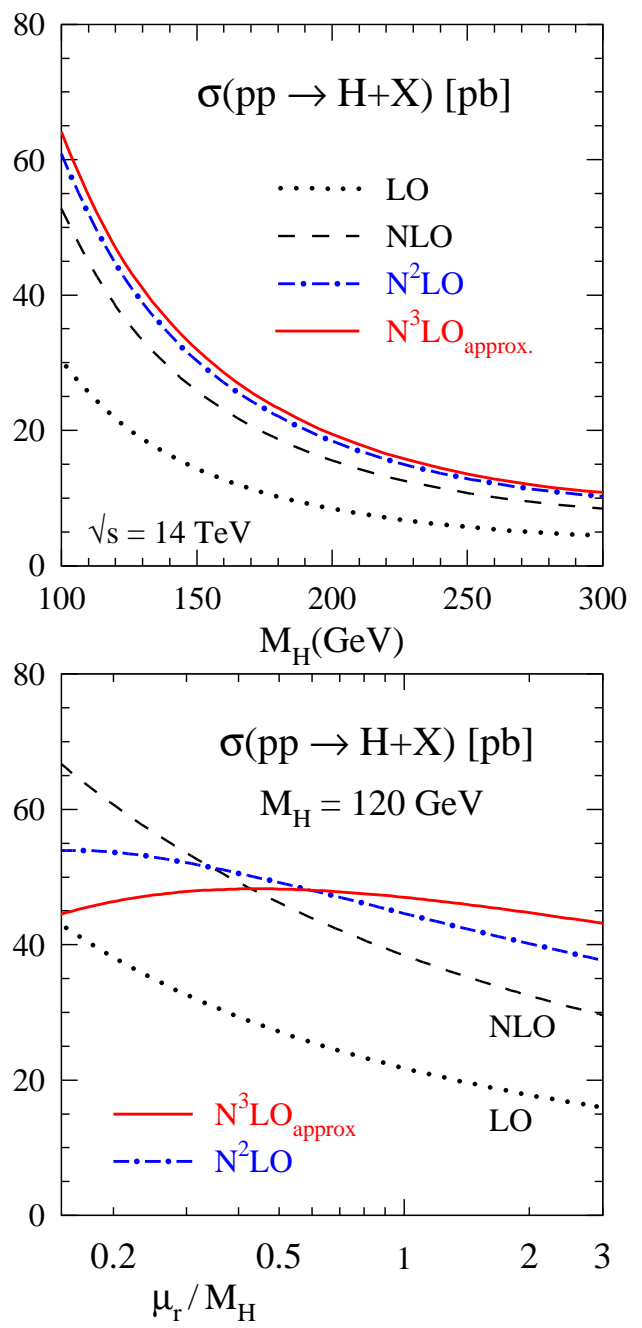

Figure 4. Perturbative expansion of the total cross section of Higgs production at the LHC for the parton densities of Refs. [48,49]. Top: dependence on the Higgs mass $M_{\mathrm{H}}$. Bottom: renormalization scale dependence for $M_{\mathrm{H}}=120 \mathrm{GeV}$.

\section{Summary}

We have computed the third-order coefficient functions for the most important structure functions in deep-inelastic scattering $[5,6,30]$. This first calculation of the three-loop corrections to one-scale partonic cross sections offers new insights into the process at hand, and facilitates improved determinations of the strong coupling constant $\alpha_{\mathrm{s}}$. Moreover it has lead to further important third-order (and all-order resummed, cf. Ref. [10,11]) results for the on-shell quark and gluon form factors $[7,8]$ and the cross section for Higgs boson production at proton colliders [9].

\section{Acknowledgments}

The work of S.M. has been supported in part by the Helmholtz Gemeinschaft under contract VH-NG-105. The work of J.V. has been part of the research program of the Dutch Foundation for Fundamental Research of Matter (FOM).

\section{REFERENCES}

1. S. Moch, J.A.M. Vermaseren and A. Vogt, Nucl. Phys. B688 (2004) 101, hep-ph/0403192

2. A. Vogt, S. Moch and J.A.M. Vermaseren, Nucl. Phys. B691 (2004) 129, hep-ph/0404111

3. A. Vogt, S. Moch and J.A.M. Vermaseren, Proceedings of DIS 2004, Strbské Pleso, Slovakia, April 2004, eds. D. Bruncko et al. (Institute of Exp. Phys. SAS, Kosice 2004), p. 231, hep-ph/0407321

4. S. Moch, J.A.M. Vermaseren and A. Vogt, Nucl. Phys. Proc. Suppl. 135 (2004) 137, hepph/0408075

5. S. Moch, J. A. M. Vermaseren and A. Vogt, Phys. Lett. B606 (2005) 123, hep-ph/0411112

6. J. A. M. Vermaseren, A. Vogt and S. Moch, Nucl. Phys. B724 (2005) 3, hep-ph/0504242

7. S. Moch, J. A. M. Vermaseren and A. Vogt, JHEP 0508 (2005) 049, hep-ph/0507039

8. S. Moch, J. A. M. Vermaseren and A. Vogt, Phys. Lett. B625 (2005) 245, hep-ph/0508055

9. S. Moch and A. Vogt, Phys. Lett. B631 (2005) 48, hep-ph/0508265 
10. S. Moch, J. A. M. Vermaseren and A. Vogt, Nucl. Phys. B726 (2005) 317, hep-ph/0506288

11. S. Moch, A. Vogt and J. Vermaseren, Acta Phys. Pol. B36 (2005) 3295, hep-ph/0511113

12. A. Vogt, S. Moch and J. Vermaseren, Acta Phys. Pol. B37 (2006) 683, hep-ph/0511112

13. A. Mitov, S. Moch and A. Vogt, Phys. Lett. B638 (2006) 61, hep-ph/0604053

14. A. Mitov and S. Moch, these proceedings

15. E. Remiddi and J.A.M. Vermaseren, Int. J. Mod. Phys. A15 (2000) 725, hep-ph/9905237

16. S. Moch and J.A.M. Vermaseren, Nucl. Phys. B573 (2000) 853, hep-ph/9912355

17. J.A.M. Vermaseren, math-ph/0010025

18. J.A.M. Vermaseren, Nucl. Phys. Proc. Suppl. 116 (2003) 343, hep-ph/0211297

19. S.G. Gorishnii et al., Comput. Phys. Commun. 55 (1989) 381

20. S.A. Larin, F.V. Tkachev and J.A.M. Vermaseren, NIKHEF-H-91-18

21. S. Larin, T. van Ritbergen, and J.A.M. Vermaseren, Nucl. Phys. B427 (1994) 40

22. S. Larin et al., Nucl. Phys. B492 (1997) 338, hep-ph/9605317

23. A. Retey and J.A.M. Vermaseren, Nucl. Phys. B604 (2001) 281, hep-ph/0007294

24. J. Blümlein and J.A.M. Vermaseren, Phys. Lett. B606 (2005) 130, hep-ph/0411111

25. A.D. Martin, W.J. Stirling and R.S. Thorne, Phys. Lett. B635 (2006) 305, hep-ph/0601247

26. W.L. van Neerven and A. Vogt, Nucl. Phys. B603 (2001) 42, hep-ph/0103123

27. P.A Baikov and K.G Chetyrkin, these proceedings

28. J. Blümlein, H. Böttcher and A. Guffanti, hep-ph/0607200

29. E.B. Zijlstra and W.L. van Neerven, Phys. Lett. B273 (1991) 476

30. J. A. M. Vermaseren, A. Vogt and S. Moch, in preparation

31. A. Vogt, Comput. Phys. Commun. 170 (2005) 65, hep-ph/0408244

32. S. Catani, Phys. Lett. B427 (1998) 161, hep$\mathrm{ph} / 9802439$
33. G. Sterman and M.E. Tejeda-Yeomans, Phys. Lett. B552 (2003) 48, hep-ph/0210130

34. K. Chetyrkin, B. Kniehl and M. Steinhauser, Nucl. Phys. B510 (1998) 61, hep-ph/9708255

35. Y. Schröder and M. Steinhauser, JHEP 0601 (2006) 051, hep-ph/0512058

36. K. G. Chetyrkin, J. H. Kühn and C. Sturm, Nucl. Phys. B744 (2006) 121, hep-ph/0512060

37. T. Matsuura and W.L. van Neerven, Z. Phys. C38 (1988) 623

38. R. Harlander, Phys. Lett. B492 (2000) 74, hep-ph/0007289

39. T. Gehrmann, T. Huber and D. Maitre, Phys. Lett. B622 (2005) 295, hep-ph/0507061

40. T. Gehrmann et al., hep-ph/0607185

41. S. Moch, J. Vermaseren and A. Vogt, Nucl. Phys. B646 (2002) 181, hep-ph/0209100

42. J.C. Collins, Phys. Rev. D22 (1980) 1478

43. A. Sen, Phys. Rev. D24 (1981) 3281

44. L. Magnea and G. Sterman, Phys. Rev. D42 (1990) 4222

45. V. Ravindran, J. Smith and W. van Neerven, Nucl. Phys. B704 (2005) 332, hep-ph/0408315

46. A.V. Kotikov et al, Phys. Lett. B595 (2004) 521, B632 (2006) 754 (E), hep-th/0404092

47. Z. Bern, L.J. Dixon and V.A. Smirnov, Phys. Rev. D72 (2005) 085001, hep-th/0505205

48. A.D. Martin et al., Eur. Phys. J. C23 (2002) 73, hep-ph/0110215

49. A.D. Martin et al., Phys. Lett. B531 (2002) 216, hep-ph/0201127

50. E. Laenen and L. Magnea, Phys. Lett. B632 (2006) 270, hep-ph/0508284

51. A. Idilbi et al., Phys. Rev. D73 (2006) 077501 , hep-ph/0509294

52. V. Ravindran, Nucl. Phys. B746 (2006) 58, hep-ph/0512249

53. R.V. Harlander and W.B. Kilgore, Phys. Rev. Lett. 88 (2002) 201801, hep-ph/0201206

54. C. Anastasiou and K. Melnikov, Nucl. Phys. B646 (2002) 220, hep-ph/0207004

55. V. Ravindran, J. Smith and W. van Neerven, Nucl. Phys. B665 (2003) 325, hep-ph/0302135 